\author{
Marquette University \\ e-Publications@Marquette
}

College of Nursing Faculty Research and

Publications

Nursing, College of

$5-1994$

\title{
Use Effectiveness of the Creighton Model Ovulation Method of Natural Family Planning
}

Richard Fehring

Marquette University, richard.fehring@marquette.edu

Donna M. Lawrence

Marquette University

Connie Philpot

Marquette University

Follow this and additional works at: https://epublications.marquette.edu/nursing_fac

Part of the Nursing Commons

\section{Recommended Citation}

Fehring, Richard; Lawrence, Donna M.; and Philpot, Connie, "Use Effectiveness of the Creighton Model

Ovulation Method of Natural Family Planning" (1994). College of Nursing Faculty Research and

Publications. 16.

https://epublications.marquette.edu/nursing_fac/16 


\title{
Use Effectiveness of the Creighton Model Ovulation Method of Natural Family Planning
}

\author{
Author: Richard J. Fehring, RN, DNSc, Donna Lawrence, RN, MSN, Connie Philpot \\ $\mathrm{RN}, \mathrm{MSN}$
}

\section{Abstract:}

Objective: To determine the use effectiveness of the Creighton model ovulation method in avoiding and achieving pregnancy.

Design: Prospective, descriptive.

Setting: A natural family planning clinic at a university nursing center.

Participants: Records and charts from 242 couples who were taught the Creighton model. The sample represented 1,793 months of use of the model.

Main outcome measure: Creighton model demographic forms and logbook.

Results: At 12 months of use, the Creighton model was $98.8 \%$ method effective and $98.0 \%$ use effective in avoiding pregnancy. It was $24.4 \%$ use effective in achieving pregnancy. The continuation rate for the sample at 12 months of use was $78.0 \%$.

Conclusion: The Creighton model is an effective method of family planning when used to avoid or achieve pregnancy. However, its effectiveness depends on its being taught by qualified teachers. The effectiveness rate of the Creighton model is based on the assumption that if couples knowingly use the female partner's days of fertility for genital intercourse, they are using the method to achieve pregnancy.

Modern methods of natural family planning have been developed only recently. John and Evelyn Billings developed the ovulation method in the 1960s and introduced it in the United States in the early 1970s. Since then, a number of variations of the method have been developed. In the mid-1970s and early 1980s, Hilgers, Daly, Hilgers, and Prebil (1982) and other researchers developed a standardized version of the ovulation method that is now called the Creighton model.

Like the Billings ovulation method, the Creighton model is based on the presence or absence of cervical mucus as the determinant of fertility. However, the Creighton model has a standard teaching format and language for classifying cervical mucus that differs from the Billings method (Hilgers, Daly, Prebil, \& Hilgers, 1992). In addition, it is taught only by natural 1 Fehring, Lawrence, \& Philpot 
family planning practitioners, who have been educated in a year-long program accredited by the American Academy of Natural Family Planning (AANFP). Standardization of the Creighton model is accomplished through the teaching format and curriculum. The model is considered one of the best methods of natural family planning in the United States because of its extensive research base, accredited teaching programs, and standardized teaching format (Hilgers, 1991).

Four studies have been undertaken on the effectiveness of the Creighton model-three to determine its method and use effectiveness in avoiding pregnancy and its use effectiveness in achieving pregnancy and one to determine its method effectiveness in achieving pregnancy with couples of normal fertility (Doud, 1985; Hilgers, Prebil, \& Daly, 1980; Hilgers et al., 1992; Howard, 1990). The current article presents the results of a study on the effectiveness of the model in avoiding pregnancy and in achieving pregnancy with couples who were taught the model in a university nursing center.

\section{Literature Review}

Kambic (1991) reported a meta-analysis of 23 published studies that have determined the use effectiveness of modern methods of natural family planning in avoiding pregnancy. The report includes studies on the use effectiveness of the Billings ovulation method, the symptothermal method, and the modified mucus method. The ovulation method depends only on changes in cervical mucus as an indicator to predict and detect ovulation, whereas the symptothermal method uses various indicators of ovulation, including changes in the cervix, cervical mucus, and basal body temperature. Like the ovulation method, the modified mucus method is based on changes in cervical mucus but has different criteria for determining fertile and infertile days (Dorairaj, 1991). Eighteen of the studies used life-table analysis to determine use effectiveness, and five used the Pearl index. The Pearl index is calculated by dividing the number of conceptions by the number of months of use of the method of family planning being analyzed and multiplying by 1,200. The result is the pregnancy rate, expressed as the number of pregnancies per 100 women during 1 year (Labbock, Klaus, \& Perez, 1991). The index is easy to calculate but provides only an approximation of a method's effectiveness.

However, life-table analysis is an advanced nonparametric technique that adjusts data entered into a study on a longitudinal basis (Norman \& Streiner, 1986). It is more accurate than the Pearl index in determining the effectiveness of family planning methods because it adjusts for the time variations resulting from clients entering and leaving a program. For example, a

2 Fehring, Lawrence, \& Philpot 
woman who becomes pregnant will leave the analysis during the pregnancy but will re-enter it after the birth of the child. As applied to family planning, life-table analysis results in two types of effectiveness in avoiding pregnancy: method effectiveness and use effectiveness (Hilgers et al., 1982). Method effectiveness is the effectiveness of a method of family planning when it is taught correctly and used according to instructions. Use effectiveness is based on the actual use of the method and includes errors made in teaching and in use. However, natural family planning is unique in that other types of effectiveness can be calculated, namely, the method and use effectiveness in achieving pregnancy with couples of normal fertility and the effectiveness in achieving pregnancy with couples having fertility problems. This article involves analyzing only the method and use effectiveness of the Creighton model in avoiding pregnancy and the use effectiveness of the model in achieving pregnancy.

In the Kambic (1991) report, the use-effectiveness pregnancy rate based on life-table analysis ranged from 2.5 to 27.9 ; that is, 2.5 to 27.9 of 100 women became pregnant during a 1 year period. In the five studies that used the Pearl index, the use-related pregnancy rate ranged from 2.0 to 4.4. This variation in effectiveness may be attributable to a number of factors, including the type of natural family planning used, how standardized the method was, how well the teachers were prepared, and when the subjects entered the studies. However, of particular interest is that the use-effectiveness rates in the studies published after 1988 generally are higher than those in the studies of the early 1980s. Kambic speculated that this was because of improved teaching and service. Kambic cautioned that the use-effectiveness rates reported are not strictly comparable because they did not all use the same methodology, assumptions, or pregnancy classifications. For the current article, comparisons will be made only with other useeffectiveness studies of the Creighton model. Because the model has a standardized methodology, pregnancy classification system, and educational program, comparisons between studies can be made.

None of the use-effectiveness studies in the Kambic report included the Creighton model. What follows is a report of a study of the effectiveness of the Creighton model in avoiding or achieving pregnancy in couples at a natural family planning clinic of a university nursing center.

\section{Methodology}

The couples participating in this prospective use-effectiveness study were all of the 323 couples who enrolled in the Marquette University Nursing Center natural family planning

3 Fehring, Lawrence, \& Philpot 
program from October 1984 to May 1992. All of the couples completed a standardized demographic form and were informed that the data would be used for evaluative purposes. Data on all of the 646 clients also were recorded by code in a logbook. The logbook included the date of the introductory session, the reproductive category of the couple, the number of follow-up sessions attended, the date of and reason for the couple's withdrawal from the program, referral codes, pregnancies that occurred since entry into the program, whether a pregnancy evaluation took place, the pregnancy classification, and the pregnancy outcome. Data from the logbook and the demographic form were used to calculate the effectiveness rates. The use-effectiveness study was reviewed by the director of the Nursing Center and the members of the Nursing Center Committee.

Of the 323 couples who enrolled in the program, 81 were omitted from the subsequent analysis. Thirty-nine were omitted because they were taught natural family planning for fertility awareness only, 25 were infertile, 16 used condoms during the fertile periods, and 1 was pregnant at the introductory session. Of the remaining 242 couples, 114 (47.1\%) of the female partners had regular cycles, $12(5 \%)$ had long cycles, 18 (7.4\%) were breastfeeding, 34 (14.1 $\%)$ were weaning, 47 (19.4\%) were discontinuing use of oral contraceptives, 8 (3.3\%) were premenopausal, and 9 (3.75\%) were postpartum but not breastfeeding. Most of the 242 female partners were Roman Catholic (80.2\%), and most were white (93.0\%), had at least a high school education (98\%), had combined yearly incomes with their husband or partner of more than $\$ 20,000$ (76.5\%), were younger than 29 years of age (55.4\%), and had three or fewer children (90.5\%). The date of entry into the study was the day they attended the introductory session and began to chart their fertility signs. The cumulative number of ordinal months of use of the Creighton model by the 242 couples was 2,284.

All of the 242 couples were taught the Creighton model of natural family planning. The model includes an introductory session, follow-up teaching sessions, evaluations, and a rigorous teacher training program (Hilgers et al., 1992). The introductory session is a 1-hour slide program that explains the anatomy and physiology behind the method and the technical aspects of charting one's dynamics of use. At the introductory session, any couple interested in pursuing the Creighton model ovulation method makes an appointment with one of the teachers for a follow-up session. Since the beginning of the Marquette University program, 66 couples attended the introductory session but did not make a follow-up appointment. The main reasons given for not entering the program were lack of interest or the decision to use another method of family planning. The 242 couples who entered the program were given a user manual, a fertility

\section{Fehring, Lawrence, \& Philpot}


chart, fertility monitoring stamps, and an appointment to meet with a natural family planning practitioner for a follow-up session in 2 weeks. They began to chart their fertility signs the next day and were asked to read the user manual.

The Creighton model requires a minimum of eight follow-up sessions in a 1-year period. The first four are every 2 weeks, the fifth is 1 month later, and the remainder are every 3 months. Each session lasts approximately 1 hour. Standardization of the sessions is accomplished through the use of a 26-page follow-up charting form, a picture dictionary of terminology and observations, a user manual, and a case management book for the natural family planning practitioner. The sessions include information and evaluations of the observation techniques; a review of the picture dictionary and definitions; a review of the fertility chart; information on the management of stress and early ovulation; information on the management of arousal and seminal fluid; instructions on vaginal hygiene; discussions of psychological concerns; graduated instructions on days of fertility and infertility; personal evaluations of satisfaction and confidence; and a summary of case management. Some of the information given is a review and additional explanation of material from the user manual, whereas other information is supplemental. Although follow-up sessions are designed to provide the couple with a gradual understanding of the method, the couple is considered a user of the method from the day of the introductory session, and effectiveness calculations begin with that day.

Special instructions are provided in follow-up sessions according to the reproductive classification of the couple. The 10 reproductive categories are regular cycle (21-38 days), long cycles (generally longer than 38 days), breastfeeding-total, breastfeeding-weaning, postpill, premenopausal, postpartum but not breastfeeding, postabortion, infertility, and pregnancy. An example of a special instruction is informing couples who are discontinuing oral contraceptive use to avoid trying to achieve pregnancy for six cycles.

Evaluation of the teaching process is integrated into every step of the model. Standardized evaluation forms are used by the couple and the teacher at the introductory sessions and the 4th, 5th, and 6th follow-up sessions. These forms include an evaluation of the introductory session, the follow-up sessions, and the teacher. At the 4th and 6th sessions, couples are evaluated on their knowledge of the model through true-or-false questions and a review of the progression of the cycle. Each follow-up session ends with an evaluation of the couple's satisfaction, confidence, and receptivity to a pregnancy. Every time a client gets pregnant-whether or not the couple was trying to achieve it-an evaluation is conducted within the first 3 months of the pregnancy. A unique aspect of the Creighton model is that evaluations

5 Fehring, Lawrence, \& Philpot 
are conducted on all pregnancies and that the evaluations are prospective. The evaluation is viewed as the essence of quality control of a method of natural family planning. The evaluation lasts approximately 1 hour. All but two of the pregnancy evaluations in the current study were conducted in person; the remaining two were conducted by telephone. After the evaluation is completed, the pregnancy is classified according to the following categories.

1. Achieving-related pregnancy. From the available information, the method was used to achieve a pregnancy and was successful.

2. Avoidance-related pregnancy. From the available information, the method was used to avoid pregnancy and the couple became pregnant.

a. Method-related. From the available information, the method was used correctly to avoid pregnancy and the couple became pregnant.

b. Use-related. From the available information, the method was used incorrectly (although it was taught correctly) to avoid pregnancy and the couple became pregnant.

c. Teaching-related. From the available information, the method was taught incorrectly (although it was used correctly) as a method of avoiding pregnancy and the couple became pregnant.

d. Using-teaching-related. A combination of used incorrectly and taught incorrectly.

3. Unresolved pregnancy. From the available information, the circumstances of the pregnancy cannot be placed into any of the preceding classifications.

All six teachers in the Marquette program completed a program that taught the Creighton model. This program, which is accredited by the AANFP, takes approximately 1 year and includes two educational phases and two supervised practicums. After completing the training program and practicing for 1 year, during which time the practitioner's case load is increased by a minimum of 10 clients, the practitioner can apply for certification through the AANFP. Two of the Marquette practitioners were certified in this manner. The AANFP is the national certification body for practitioners who teach the Creighton model. AANFP certification signifies that the practitioner can provide quality education in the Creighton model.

We used life-table techniques, as described by Hilgers (1984), to conduct statistical analysis to determine the effectiveness of the Creighton model in avoiding pregnancy. According to Hilgers, this type of analysis loses significance in determining use-effectiveness 6 Fehring, Lawrence, \& Philpot 
once the number of couple months of use (of the method) drops below 100.0.

\section{Results}

There were 65 pregnancies among the 242 couples during a 12-month period. Table 1 lists the pregnancy rates by the Creighton model pregnancy classification and ordinal month of use. Most of the pregnancies were achieving related. The method-effectiveness rates of avoiding pregnancy (per 100 couples) were $0.0(100.0 \%)$ at the 1st ordinal month of use, 0.4 $(99.6 \%)$ at the 6th ordinal month, and $1.2(98.8 \%)$ at 12 months. The use-effectiveness rates of avoiding pregnancy (per 100 couples) were $0.0(100.0 \%)$ at the 1 st ordinal month of use, 1.2 $(98.8 \%)$ at the 6th ordinal month, and $2.0(98.0 \%)$ at the 12th ordinal month. The useeffectiveness rates of achieving pregnancy (per 100 couples) were 13.6 at 6 months of use and 24.4 at 12 months of use.

At the 12 th ordinal month, there was a $20.2 \%$ cumulative discontinuation rate among the 242 participating couples. Most of the couples who discontinued use did so for various reasons, including for personal reasons $(17.4 \%)$, to use artificial methods (1.2\%), be- cause they were having difficulty avoiding genital contact $(0.8 \%)$, because they switched to another natural method $(0.4 \%)$, and because they lacked confidence in the method $(0.4 \%)$. The estimated continuation rate for couples who became pregnant for any reason was $78.0 \%$ at the 12 th ordinal month.

\section{Discussion}

The method- and use-effectiveness rates for avoiding pregnancy in the current study are similar to those obtained in three previous studies that examined the effectiveness of the Creighton model (Doud, 1985; Hilgers et al., 1980; Howard, 1990). At the 12th ordinal month, the method effectiveness of the model for avoiding pregnancy ranged from a low of 98.8 in the current study to a high of 99.9 in the Howard study. In Hilger's study, it was 99.6 and in Doud's study 99.1. The lower method effectiveness in the current study may be attributable to some of the practitioners improperly classifying unresolved pregnancies as method related. Use-effective rates at the 12th ordinal month were 94.8 in the Hilgers et al. (1980) study, 96.2 in the Doud study, 97.4 in the Howard study, and 98.0 in the current study.

The more recent studies show a higher use-effectiveness rate in avoiding pregnancy. This is as expected because, as the years go by, the teacher education programs have improved, the model has been refined, and the practitioners have become better at teaching the 7 Fehring, Lawrence, \& Philpot 
method. This is a phenomenon similar to what Kambic (1991) found in his meta-analysis of the use-effectiveness rates of other methods of natural family planning. We conclude that the Creighton model is an effective method of family planning and is becoming more effective.

According to Hilgers et al. (1992), the use-effectiveness rate of a method of natural family planning in achieving pregnancy is the percentage per 100 couples who, during a 1-year period, decide to stop using the method to avoid pregnancy, adopt the method to achieve pregnancy, and are successful in doing so. He estimated that 1 of 5 couples during the period of a year will stop using the method to avoid pregnancy and adopt it to achieve pregnancy. Approximately 4 of 5 of those women will become pregnant. The use-effectiveness rates for achieving pregnancy among the four Creighton model studies were similar at the 12th ordinal month except for the Howard study. The Hilgers et al. (1980) study was 21.3, the 1985 Doud study 28.0, the Howard study 13.1, and the current study 24.4. However, the method effectiveness for achieving pregnancy is the effectiveness of the method of natural family planning in achieving pregnancy during a given menstrual cycle with couples of normal fertility (Hilgers et al., 1992). Hilgers et al. (1992) demonstrated that 38 of 50 (76\%) couples who used the Creighton model to achieve pregnancy did so during the first cycle.

A common misinterpretation of the Creighton model occurs regarding the determination of its use-effectiveness rates in avoiding pregnancy. Some have interpreted the model's pregnancy-achieving rates to mean that anytime a couple has intercourse during the woman's fertile period, it is classified as an achieving-related pregnancy (Hilgers, 1979; Kambic, 1991). However, this is not the case. If a couple erred in using the method, if the practitioner taught the method improperly, or if a combination of both occurred (even though the couple had intercourse during the woman's fertile period) the pregnancy would not be classified as achieving-related. If the couple was using the Creighton model to avoid pregnancy and knowingly had genital intercourse on a fertile day and the female partner became pregnant, the pregnancy would be classified as achieving-related. With the Creighton model, each couple is informed at the introductory session (with reinforcement at the follow-up sessions) that both partners will know on a given day whether the woman is fertile and that if they choose to have intercourse on that day, they have abandoned the model as a method of avoiding pregnancy and adopted it as a method of achieving pregnancy. Thus, the achieving-related classification is based on the objective behavior of the couple and not on subjective considerations of whether or not a pregnancy is wanted.

In interpreting the use-effectiveness data of the Creighton model, one should remember 8 Fehring, Lawrence, \& Philpot 
that the model is not considered a method of contraception. Natural family planning can be used to achieve or to avoid pregnancy. A couple can choose to use the method to achieve or avoid pregnancy or not to use the method at all. If the couple knows that the female partner is fertile on a given day of the cycle and decides to have intercourse, that is the couple's choice. By contrast, with contraception, one can either use the method to avoid pregnancy or not use it at all. Because natural family planning differs from contraception, different interpretations are needed for classifying pregnancies.

A problem in the interpretation of use-effectiveness rates in other contraceptive and natural family planning studies is that their descriptions of behaviors and classification are unclear (Hilgers, 1979). Vague references are made to wanted or unwanted pregnancies, taking chances, failure to follow rules, surprise pregnancies, and perfect and imperfect use of the method (Hilgers, 1979; Trussell \& Grummer-Strawn, 1991). No universally accepted criteria exist for classifying pregnancies. The Creighton model provides a classification that is based on the objective behavior of the couple.

In contrast, most researchers of use-effectiveness rates of natural family planning report pregnancies that result when couples knowingly abandon the method for avoiding pregnancy, and they classify such pregnancies as use-related, rather than achieving-related. Thus, such pregnancies are calculated into the use-effectiveness rates of the given method of family planning. For example, the World Health Organization (WHO) reported a five-country study of the ovulation method in 1981. The report's use-effectiveness rates in avoiding pregnancy, based on a modified Pearl index, ranged from 13.8 to 33.6, with an average of 22.5. However, an average of $15.4 \%$ of the couples "consciously departed from the rules" (i.e., the pair had intercourse on days when the woman was fertile, although both partners knew the woman was fertile), and the women became pregnant. If these pregnancies were classified as achievingrelated pregnancies, the effectiveness rate would be 7.1 , or $92.9 \%$ effective in avoiding pregnancy. If the WHO interpretation were used with the current study, the use-effectiveness rate in avoiding pregnancy would be 9.9 at the 6th ordinal month and 12.8 at the 12th month. However, this interpretation is tenuous at best because the couples in the study did not abandon any rules (they knew the instructions and the days of fertility and infertility); rather, they chose to use the method to achieve pregnancy. When the couples were questioned about their behavior, they did not refer to it as abandoning the rules or taking a chance but indicated that they knew they could achieve a pregnancy.

Additional research is needed to determine the use effectiveness of the Creighton model

9 Fehring, Lawrence, \& Philpot 
and other methods of natural family planning with women of various reproductive categories. For example, how effective is the Creighton model with women who are breastfeeding or premenopausal? Larger, multicenter studies will be needed for this type of research, to obtain large enough samples for special reproductive categories. A limitation of the current study is its relatively small, homogeneous sample. Most of the couples were college educated, white, and Roman Catholic. Additional research on the use effectiveness of the Creighton model should include larger numbers of people from more diverse populations. A final area for additional research is the decision-making processes and behaviors that couples use when planning a pregnancy. This type of study probably would be best conducted through a qualitative approach. The answers might help determine objective measures of when couples want or do not want to achieve a pregnancy.

\section{Nursing Implications}

The current study has shown that nurses who use, recommend, or teach couples the Creighton model can be confident that the model is an effective method of family planning when used to avoid pregnancy. Nurses also should be mindful that the model is based on the assumption that when couples use its methodology, they will know on any given day whether the female partner is fertile, and if they knowingly have genital intercourse on days when she is fertile, they are, in effect, abandoning the method as a method of avoiding pregnancy and adopting it to achieve pregnancy.

Another implication of the study is that nurses should be aware of the basic assumptions underlying the notion of the use effectiveness of natural family planning methods. Hilgers (1984) recommends that a number of essential quality-control prerequisites need to be in place before a use-effectiveness study can be conducted. These prerequisites can be used by a nurse to judge the use effectiveness of a given method of natural family planning. The prerequisites are (1) that the method have short-and long-term follow-up sessions, (2) that the teaching methodology be standardized, (3) that pregnancy evaluations be conducted in a prospective fashion and with a standardized format, (4) that the study include clients of all reproductive categories except known infertility, and (5) that the clients be exposed from the beginning to the idea that the method can be used to achieve or avoid pregnancy. Among the prerequisites recommended by other researchers are that the educational materials of the method be standardized, that there be objective assessments of the client's learning progress, that each client have access to method-specific information, and that the educational curriculum and

10 Fehring, Lawrence, \& Philpot 
overall qualifications of the teachers be included in the study of use effectiveness. These prerequisites are quality standards that nurses are advised to look for in a natural family planning program. These quality standards are part of the Creighton model.

\section{References}

Dorairaj, K. (1991). The modified mucus method in India. American Journal of Obstetrics and Gynecology, 165(Suppl), 2066-2067.

Doud, J. (1985). Use-effectiveness of the Creighton model of NFP. International Review of Natural Family Planning, 9, 54-72.

Hilgers, T. W. (1979). A critical evaluation of effectiveness studies in natural family planning. In Proceedings of an International Symposium on Natural Family Planning Sponsored by the World Health Organization (pp. 94-109). Department of Health, Dublin, Ireland.

Hilgers, T. W. (1984). The statistical evaluation of natural family planning. International Review of Natural Family Planning, 8, 226-264.

Hilgers, T. W. (1991). The medical applications of natural family planning. Omaha, NE: Pope Paul VI Institute Press.

Hilgers, T. W., Daly, K. D., Hilgers, S. K., \& Prebil, A. M. (1982). The ovulation method of natural family planning: A standardized case management approach to teaching. Book I. Omaha, NE: Creighton University Natural Family Planning Education and Research Center.

Hilgers, T. W., Daly, K. D., Prebil, A. M., \& Hilgers, S. K. (1992). Cumulative pregnancy rates in patients with apparently normal fertility and fertility-focused intercourse. Journal of Reproductive Medicine, 37(10), 864-866.

Hilgers, T. W., Prebil, A. M., \& Daly, K. D. (1980, July). The effectiveness of the ovulation method as a means of achieving and avoiding pregnancy. Paper presented at the Education Phase III Continuing Education Conference for NFP Practitioners, Mercy Fontenelle Center, Omaha, Nebraska.

Howard, M. P. (1990). Use-effectiveness of the ovulation method (Creighton model) of natural family planning, St. Joseph Hospital, Houston, Texas. Paper presented at the Ninth Annual Meeting of the American Academy of Natural Family Planning, Milwaukee, Wisconsin.

Kambic, R. T. (1991). Natural family planning use-effectiveness and continuation. American Journal of Obstetrics and Gynecology, 165(Suppl), 2046-2048.

11 Fehring, Lawrence, \& Philpot 
Labbock, M. H., Klaus, H., \& Perez, A. (1991). Efficacy studies in natural family planning: Issues and management implications illustrated with data from five studies. American Journal of Obstetrics and Gynecology, 165(Suppl), 2048-2051.

Norman, G., \& Streiner, D. (1986). PDO statistics. Philadelphia: B. C. Decker, Inc.

Trussell, J., \& Grummer-Strawn, L. (1991). Further analysis of contraceptive failure of the ovulation method. American Journal of Obstetrics and Gynecology, 154(Suppl), 20542059.

World Health Organization. (1981). A prospective multicentre trial of the ovulation method of natural family planning: II. The effectiveness phase. Fertility and Sterility, 86, 591-598.

\section{Notes}

Address for correspondence: Richard J. Fehring, RN, DNSc, Associate Professor, Marquette University College of Nursing, Milwaukee, WI 53233.

Richard J. Fehring is an associate professor at the Marquette University College of Nursing and the coordinator of the Marquette University Natural Family Planning Services, Milwaukee, WI.

Donna Lawrence is an associate professor at the Marquette University College of Nursing and a certified Natural Family Planning practitioner at Marquette University's Nursing Center, Milwaukee, WI.

Connie Philpot is a research assistant at the Marquette University College of Nursing, Milwaukee, WI.

\section{Appendix}

Table 1

Cumulative Pregnancy Rates by Pregnancy Classification and Ordinal Month of Use*

\begin{tabular}{|c|c|c|c|c|c|c|c|}
\hline & \multicolumn{7}{|c|}{ Pregnancy Classification } \\
\hline $\begin{array}{c}\text { Ordinal } \\
\text { Month }\end{array}$ & Total & $\mathbf{1}$ & $\mathbf{2 a}$ & $\mathbf{2 b}$ & $\mathbf{2 c}$ & $\mathbf{2 d}$ & $\mathbf{3}$ \\
\hline 1 & 1.2 & 1.2 & 0 & 0 & 0 & 0 & 0 \\
\hline 6 & 15.2 & 13.6 & 0.4 & 0.8 & 0 & 0 & 0.4 \\
\hline 12 & 26.8 & 24.4 & 1.2 & 0.8 & 0 & 0 & 0.4 \\
\hline
\end{tabular}

Based on 1,792 ordinal months of use at the 12th ordinal month.

12 Fehring, Lawrence, \& Philpot 